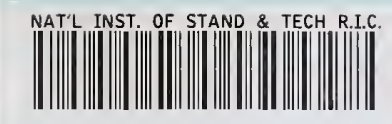

All105 102344
REFERENCE

52

NISTIR 5927

\title{
On the Translation of Kif/Frame Ontologies to EXPRESS
}

Peter R. Wilson

Catholic Univeristy of America

U.S. DEPARTMENT OF COMMERCE Technology Administration National Institute of Standards and Technology

Gaithersburg, MD 20899-0001

$Q C$

100

.456

No. 5957

1996

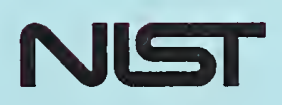





\section{On the Translation of Kif/Frame Ontologies to EXPRESS}

\section{Peter R. Wilson}

Catholic Univeristy of America

U.S. DEPARTMENT OF COMMERCE

Technology Administration

National Institute of Standards and Technology

Gaithersburg, MD 20899-0001

August 1996

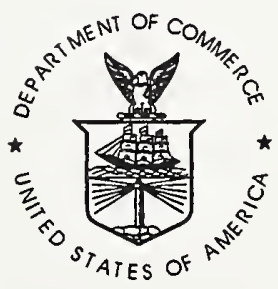

U.S. DEPARTMENT OF COMMERCE Michael Kantor, Secretary

TECHNOLOGY ADMINISTRATION

Mary L. Good, Under Secretary for Technology

NATIONAL INSTITUTE OF STANDARDS

AND TECHNOLOGY

Arati Prabhakar, Director 



\title{
On the Translation of Kif/Frame Ontologies to EXPRESS
}

\author{
Peter R. Wilson \\ Catholic University of America* \\ pwilsonQcme.nist.gov
}

August 1996

\begin{abstract}
This report describes the translation of three groups of ontologies specified using the Frame variant of the Knowledge Interchange Format (KIF) language into information models specified using the EXPRESS information modeling language, as defined in International Standard ISO 10303-11:1994. This work was undertaken to better understand the relationship between KIF/Frame and EXPRESS. From the work done to date the capabilities of KIF/Frame and EXPRESS appear to be broadly similar but they differ in detail.
\end{abstract}

\section{Contents}

1 Introduction 1

2 An overview of the translation process 1

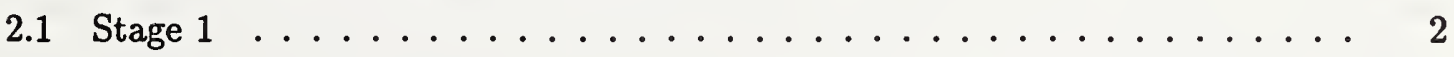

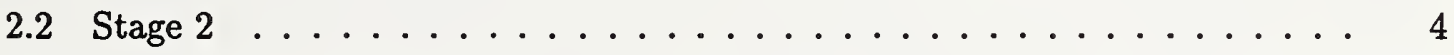

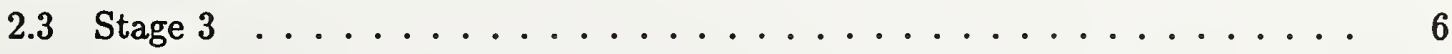

3 Observations $\quad 9$

3.1 Theory vs. Schema . . . . . . . . . . . . . . . . 9

3.2 Classes, Relations, Entities and such . . . . . . . . . . . . 10

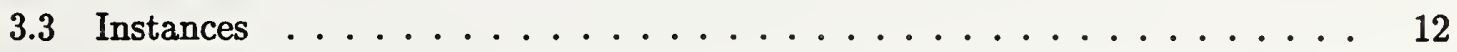

4 Basic elements of Frame and EXPRESS

- This work was performed as a Guest Researcher at the National Institute of Standards and Technology. 
A EXPRESS example model $\quad 16$

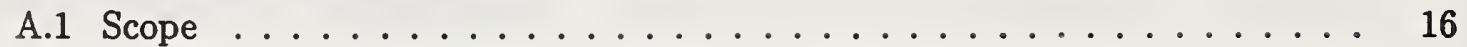

A.2 Model overview ......................... 16

A.3 Authority schema . . . . . . . . . . . . . . . 17

A.3.1 Entity definitions .......................... 17

A.3.2 Function and procedure definitions . . . . . . . . . . 20

A.3.3 Entity classification structure ................ 23

A.4 Support schema . . . . . . . . . . . . . . . . . 23

A.4.1 Type definitions ......................... 23

A.4.2 Entity definitions . . . . . . . . . . . . . . . 24

A.4.3 Function and procedure definitions . . . . . . . . . . 32

A.4.4 Entity classification structure . . . . . . . . . . . 34

A.5 Calendar schema . . . . . . . . . . . . . . . . . . . 34

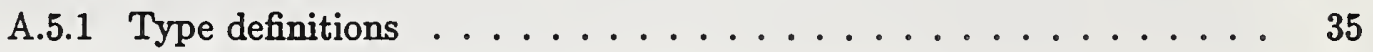

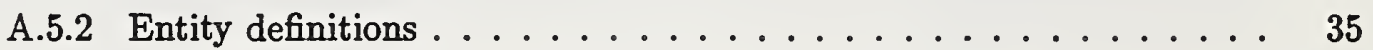

A.5.3 Function and procedure definitions . . . . . . . . . . 36

A.5.4 Entity classification structure . . . . . . . . . . . . 38 


\section{Introduction}

The Knowledge Interchange Format (KIF [Gin91], [GF92]) is being proposed as an ANSI Standard [X395]. There is another language, namely EXPRESS, which has recently become an ISO International Standard [SW94], [ISO94]. Both these languages are intended to enable the precise and formal modeling of information. An exercise was undertaken to translate some of the KIF-defined ontologies into EXPRESS in order to try and determine whether this was possible. In particular, several ontologies were translated from versions described using the automatically generated generic frame representations.

This document reports on the general results from this exercise. The Ontologies used were specified in a generic Frame language that had been generated automatically from the original KIF specification. The ontologies were obtained in December 1995 from: http://ww-ksl.stanford.edu/knowledge-sharing/ontologies.

The EXPRESS renditions of the selected ontologies are given elsewhere [Wil96a], [Wil96b], [Wil96d]. It is assumed that the reader has some knowledge of both KIF (and the corresponding frame language) and the EXPRESS language.

Section 2 provides an overview of the processes used to translate from the KIF/Frame ontologies into EXPRESS models. Some observations resulting from this are given in Section 3. The next section (4) discusses the basic elements of Frame and EXPRESS and notes their similarities and differences. Conclusions are given in Section 5. Finally, an EXPRESS model is given in Appendix A as a challenge for KIF/Frame experts to translate into an ontology.

\section{An overview of the translation process}

No language exactly maps one-for-one into another language. This, though, does not necessarily imply the languages are not equally expressive. There are two forms of translation, one that I call transliteration and the other called idiomatic. In the transliteration case the result looks or sounds strange to a native speaker. Let us take the French sentence Il pleut comme une vache! as an example. A transliteration of this into English is: 'It is raining like a cow!'. On the other hand, an idiomatic translation will probably result in 'It is raining cats and dogs!'. In turn, a transliteration of this back into French would puzzle most French speakers.

Essentially, the goal of a good translation is to end with an idiomatic rather than a transliterated result.

Briefly, the translation from the frame language to EXPRESS was done in the following manner. 


\section{$2.1 \quad$ Stage 1}

This was a fairly mechanical process.

1. For each frame theory, create a similarly named EXPRESS Schema.

2. For each frame construct of the form define-frame NAME, create an EXPRESS Entity called Name. The name translation scheme used was:

- Change NAME to Name.

- Change FIRST-SECOND to FirstSecond.

- Change FIRST.SECOND to FirstAndSEcond.

Where the frame has an own-slot of kind SUBCLASS-OF NAME, or similar, make the EXPRESS entity a SUBTYPE OF (Name). That is, map the frame SUBCLASS-OF into an EXPRESS subtype.

Where the frame has an own-slot of kind SUBCLASS-PARTITION (set of NAME1 NAME2), or similar, make the EXPRESS entity corresponding to the frame a SUPERTYPE OF (ONEOF (Name1, Name2)).

Where a frame has a template-slot, make this an attribute of the EXPRESS entity. Incorporate the simpler frame axioms into the EXPRESS model.

Some examples of these rules are:

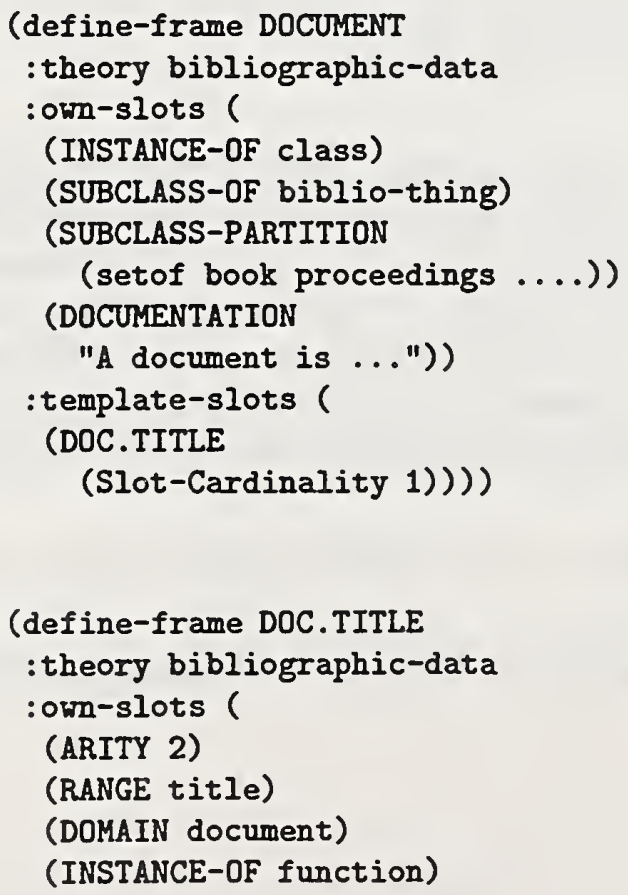

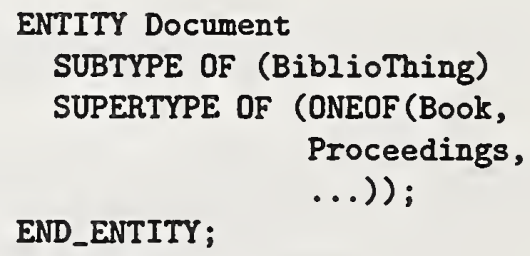




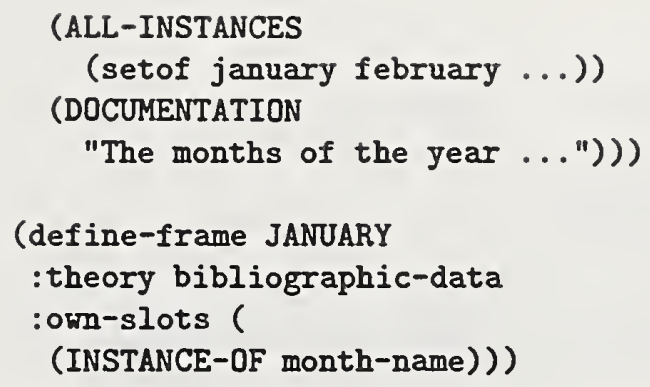

This process resulted in transliterated EXPRESS models that captured most of the intent of the ontologies translated. However, the models were not complete at this point and requires further work; principally formulating the frame axioms in terms of EXPRESS constructs and constraint language.

\section{$2.2 \quad$ Stage 2}

This stage is intended to complete the Stage 1 EXPRESS model.

1. Some axioms are of the form shown in the frame definition below, which has been taken from a bibliographic ontology:

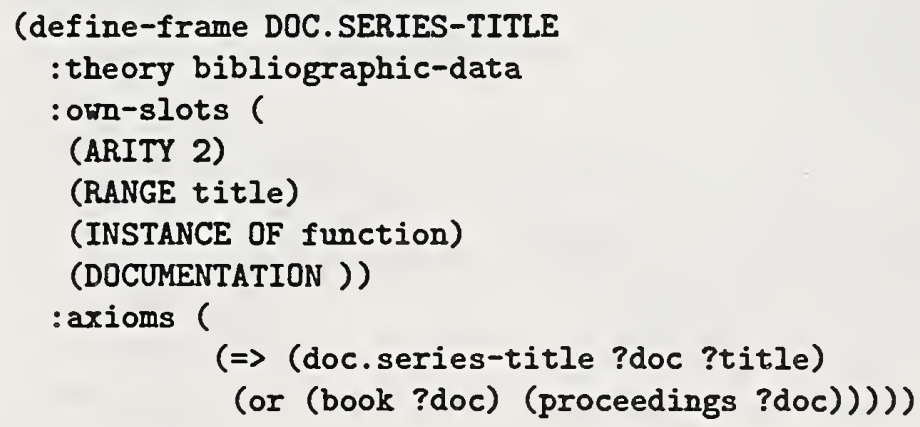

This specification is basically saying that only a Book or a Proceedings (which are two among several kinds (subtypes) of Document) can have a Series-Title. These kinds of axioms were translated into WHERE rules specifying the required type restrictions. For example, the above frame could be translated into:

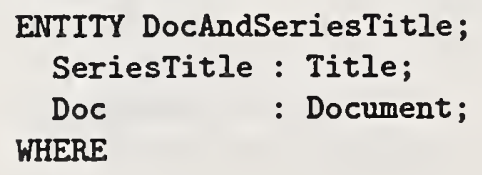


wr1 : ('BIBLIO.BOOK' IN TYPEOF(DOC)) OR

('BIBLIO.PROCEEDINGS' IN TYPEOF (DOC));

END_ENTITY;

2. Change binary relations to (optional and/or list) attributes.

The model resulting from Stage 1 has many entities that look like binary relationships.

That is, there are entities Ent1, Ent2 and BinRel where BinRel is like:

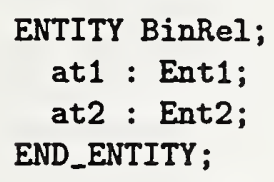

For a more idiomatic translation these BinRel are candidates for replacement by attributes of appropriate cardinality in either Ent1 or Ent2 like:

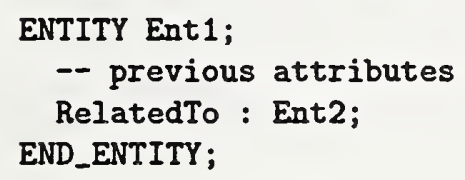

Taking the specification above of DocAndSeriesTitle as a concrete example, this could be reconfigured as:

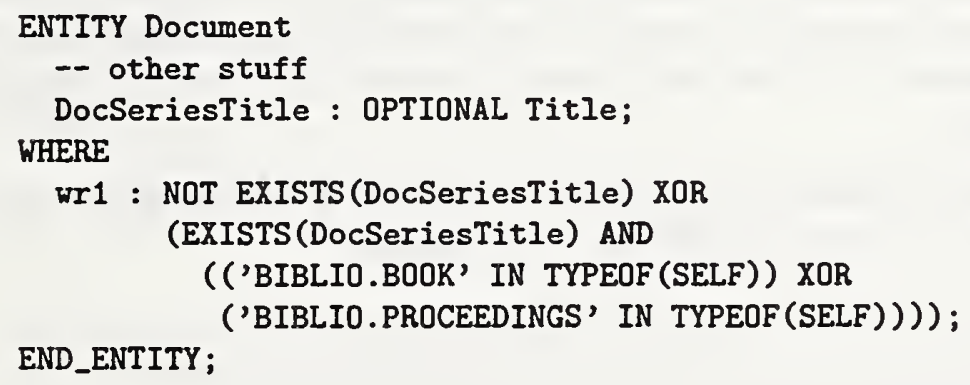

3. In many cases value-related axioms in the frame model could be translated into derived attributes in the EXPRESS model.

For example:

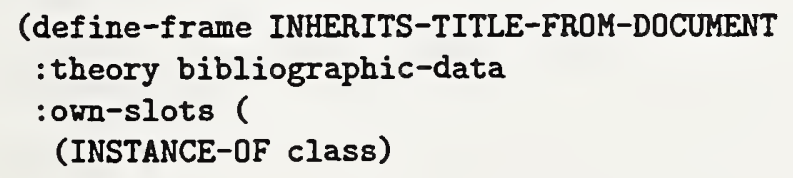




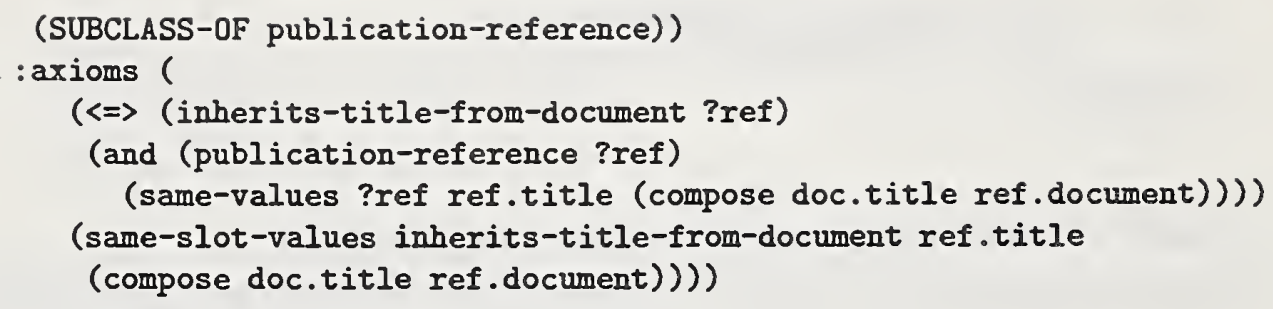

can be translated into

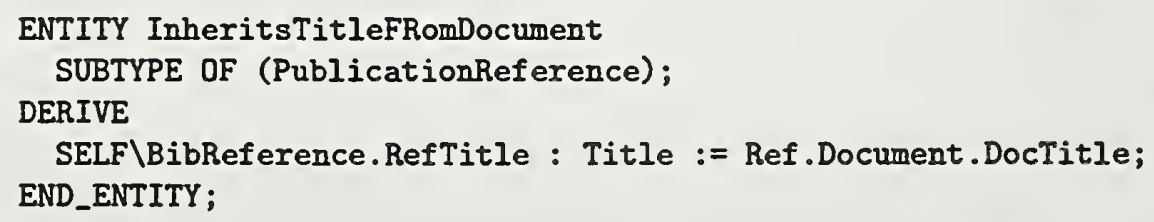

\subsection{Stage 3}

This stage is basically tidying up the EXPRESS model and presenting it in an idiomatic manner.

1. Add in any elements missing from the Frame model.

For example, one part of the bibliographic ontology dealt with the referencing of a paper that had been published in a printed proceedings. Data fields were defined for the author, title, and so on of the paper but the means of referencing the proceedings themselves was missing. In all fairness, though, the ontologies were not claimed to be necessarily complete.

2. In some cases binary relations were converted into EXPRESS functions called from derived attributes. For instance, consider the following:

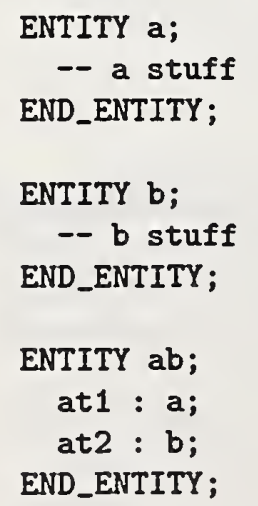


This can be written as:

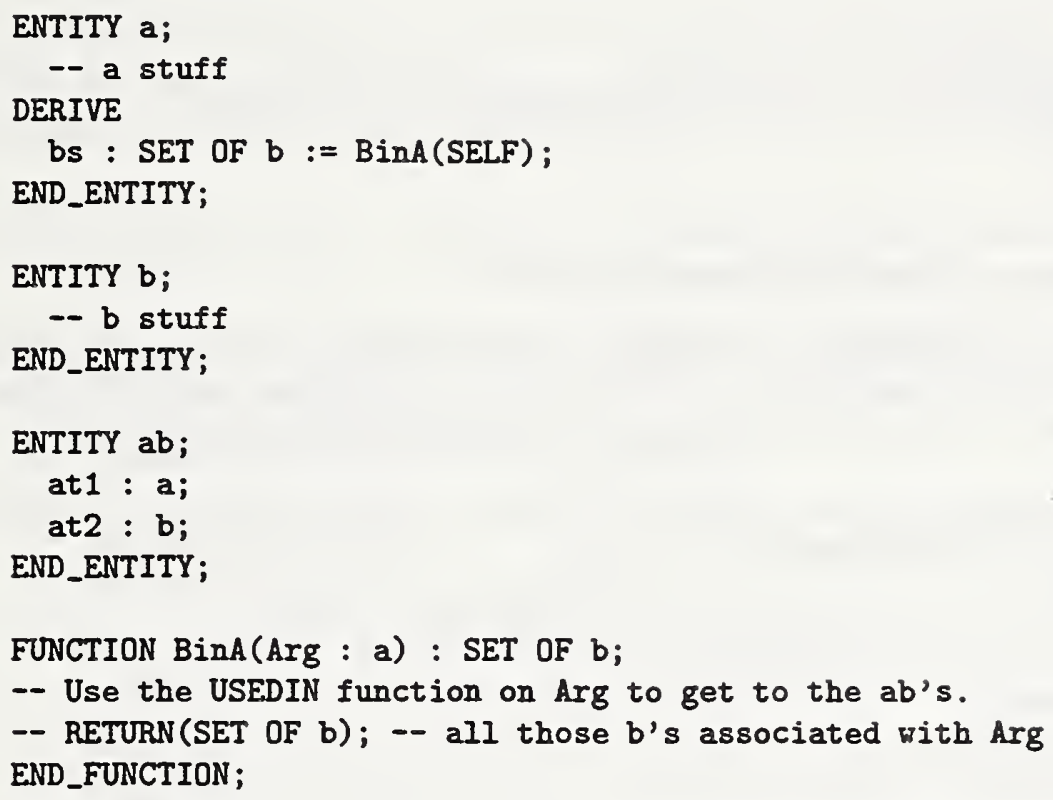

This is at first sight not a particluarly useful transformation. However, there were cases in the Frame ontologies that, after translation into EXPRESS appeared like:

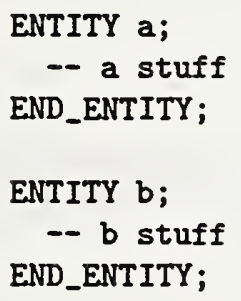

with a an axiom concerning some relationship between a's and b's, although though was no frame specifying the relationship - this was missing from the ontology. For instance, such an axiom might be that no more than three instances of $b$ could be associated with each instance of a. This could be dealt with in the EXPRESS model by introducing an appropriate function (call).

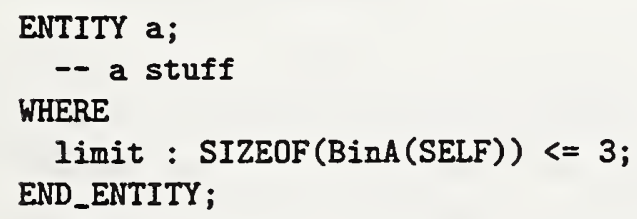

ENTITY b; 
-- b stuff

END_ENTITY;

FUNCTION BINA(Arg : a) : SET OF b;

-- RETURN(SET OF b); -- all those b's associated with Arg

END_FUNCTION;

3. Remove synonyms from the EXPRESS model.

Again taking the bibliographic ontology as an example, in some places the publisher was referred to as an 'institution', in others as an 'organization' and in yet other places as a 'publisher'. All these were collapsed in the single identifier 'publisher'.

4. Revise the EXPRESS model to convert, as far as possible, logical (procedural) constraints into the model structure.

As an example, recall the constraints in the Document entity, i.e.,

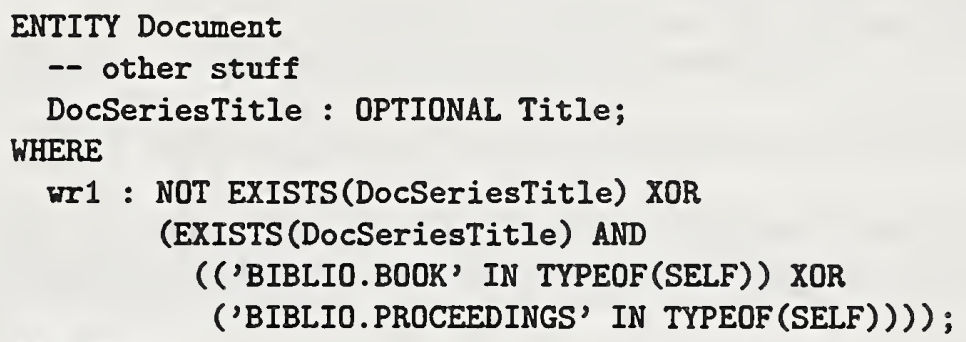

Checking the validity of an instance of Document requires the evaluation of the logical statement in the WHERE clause. This can instead be modeled structurally as:

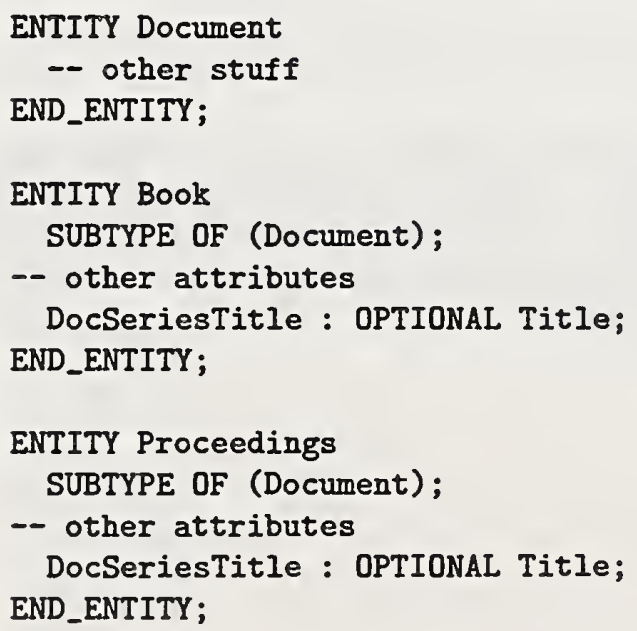




\section{Observations}

Three groups of ontologies were firstly transliterated into EXPRESS models and then massaged into idiomatic EXPRESS. In some cases a group required the conversion of more than one ontology. The grouping and ontologies were:

1. bibliographic-data - the scope is bibliographic references, such as might appear at the end of a technical or scholarly document [Wil96a].

2. constraints - a general ontology describing the specification of constraints in the form of logical sentences [Wil96b].

Some other ontologies were extensions of this.

- component-assemblies - a general ontology about assemblies of things, including sub-assemblies and connections.

- components-with-constraints - an ontology about components (from component-assemblies that have constraints (from constraints).

- mechanical-components - an ontology specializing component-assemblies to assemblies of mechanical things.

3. frame-ontology - an ontology describing the generic frame language [Wil96d].

This called on two other ontologies:

- kif-relations - an ontology describing relationships among objects.

- kif-sets - an ontology delaing with set theory.

These were all obtained in December 1995 from http://www-ksl. stanford.edu/knowledge-sharing/ontologies.

Table 1 gives a comparison between the sizes of the different models in both the Frame and EXPRESS renditions. The letters at the top of the columns indicate the different ontologies.

Generally speaking, the EXPRESS models are similar or smaller in terms of the number of definitions with respect to the Frame models.

\subsection{Theory vs. Schema}

The Frame concept of Theory maps into the EXPRESS concept of Schema. Syntactically, a Theory and a Schema are treated differently. EXPRESS uses an embedded syntax so that the contents of a Schema are syntactically embedded between the constructs BEGIN_SCHEMA name; and END_SCHEMA;. The Frame language uses a referential syntax so that a construct identifies which Theory it is a member of. 
Table 1: Statistical model comparisons

\begin{tabular}{|l|rrr|r||l|rrr|r|}
\hline \multicolumn{9}{|c||}{ Frame } & \multicolumn{5}{|c|}{ EXPRESS } \\
\hline & B & CCA & F & Tot & & B & CCA & F & Tot \\
\hline Theories & 1 & 4 & 3 & 8 & Schemas & 1 & 6 & 4 & 11 \\
Misc & & 1 & 6 & 7 & Types & 6 & 4 & 3 & 13 \\
Classes & 66 & 19 & 40 & 125 & Entities & 69 & 37 & 117 & 223 \\
Functions & 40 & 6 & 27 & 73 & Functions & 3 & 10 & 1 & 14 \\
Relations & 19 & 15 & 41 & 75 & Rules & & 1 & & 1 \\
Instances & 12 & & & 12 & & & & & \\
\hline TOTAL & 137 & 45 & 117 & 299 & TOTAL & 79 & 58 & 125 & 262 \\
\hline
\end{tabular}

Both embedded and referential syntaxes have their positive and negative sides, paticularly when it comes to the means of extending a model, but this is not the place for a discussion of that point. However, I did find significant problems with the ontologies as presented in that there were things mentioned in one Theory that were not specified within that Theory (but which were presumably defined in another Theory). There was no indication in the Frame representation of where or what these (presumed) Theories were. This was particularly evident in the Constraint related Ontologies. The Schema construct in EXPRESS provides a scoping mechanism and there is a matching capability of formally specifying where specifications utilised from other Schemas are to be found. ${ }^{1}$

It thus appears that EXPRESS formally requires a model to be complete but I did not find this to be a formal requirement of the Frame specifications. (Neither did I find anything in any of the specific Frame Ontology documents to help in identifying missing specifications or their possible locations. The documentation style used for EXPRESS models in ISO 10303 specifically requires that the collection of Schemas forming a model be identified in the informal documentation as well as the EXPRESS language requirement.)

\subsection{Classes, Relations, Entities and such}

EXPRESS essentially has three constructs - Schema, Entity and Rule. In this I am including a Type as being a kind of Entity and a Function as one means of specifying a constraint that might appear in an Entity or a Rule.

The Frame ontologies use more constructs. In the studied ontologies these are typically Class, Function, Relation, Instance, and of course Theory. These constructs, except for Theory essentially have to be mapped into the EXPRESS notion of Entity. Conceptually this is no problem as EXPRESS effectively defines an Entity as 'a representation of something of interest'. In practice, there can be some difficulties.

\footnotetext{
${ }^{1}$ In fact this is a requirement of the language.
} 
The mapping of Class to Entity is reasonably straightforward as I believe that Class and Entity are virtually synonymous.

Relation also maps well to Entity. When EXPRESS was being developed there were many discussions on explicitly distinguishing between 'entity' and 'relation' as in the EntityRelationship modeling paradigm. However the decision was made to just have Entity as the more one looked at 'relationship' the more it looked and behaved like 'entity'. The Frame Relation then can be transliterated to the EXPRESS Entity. In the limited translation experience to date most of the Relations were binary relations with no attributes. Whenever one Entity references another Entity, EXPRESS automatically considers this to be a 'relationship'. It is rare to see unconstrained and unattributed 'binary relations' in EXPRESS models - these are typically modeled by a reference from one or other of the Entities concerned to the other. Thus, in many cases a Frame Relation eventually got translated into an attribute of an EXPRESS Entity.

A Frame Function has little in common with an EXPRESS Function except for the name and the general definition of a function as a map from a domain to a range. For example, the following could be a typical EXPRESS Function used to describe the combinations of days, months and years that denote a valid date.

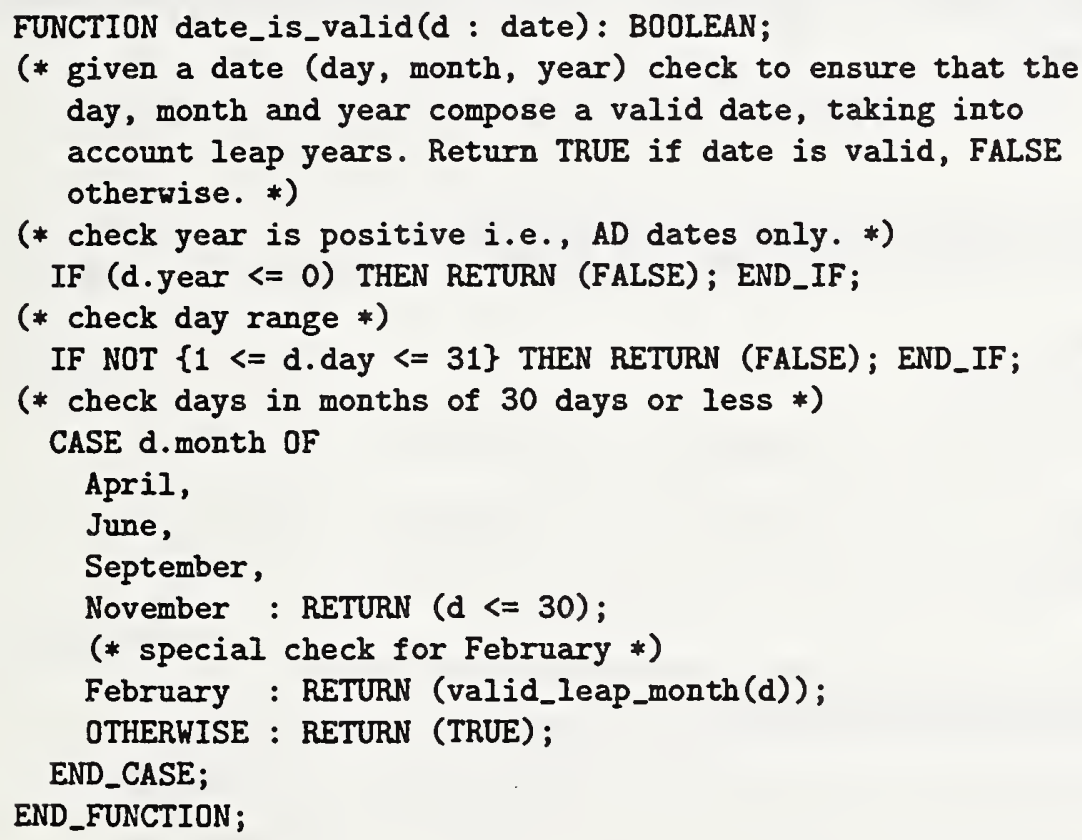

On the other hand, here is a typical Frame function:

(define-frame REF.YEAR

:theory bibliographic-data

:own-slots (

(ARITY 2) 
(RANGE year-number)

(DOMAIN reference)

(INSTANCE-OF function)

(DOCUMENTATION

"The year field is a function from a reference to the year in which the publication was published.")))

Functions like this are modeled in EXPRESS as Entity attributes. For example:

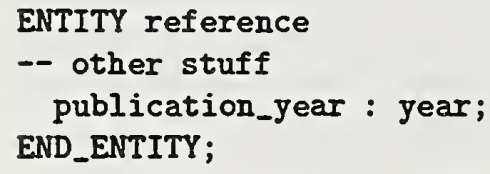

From studying the Frame ontology it appears as though the notions of Class and Function are basically two different aspects of the notion Relation. A Relation has one or more arguments. A Class is a unary Relation (a Relation with only one argument). A Function is a Relation where the 'value' of the last argument is completely determined by the preceeding arguments.

\subsection{Instances}

The Frame language has a notion of 'instances'. As examples:

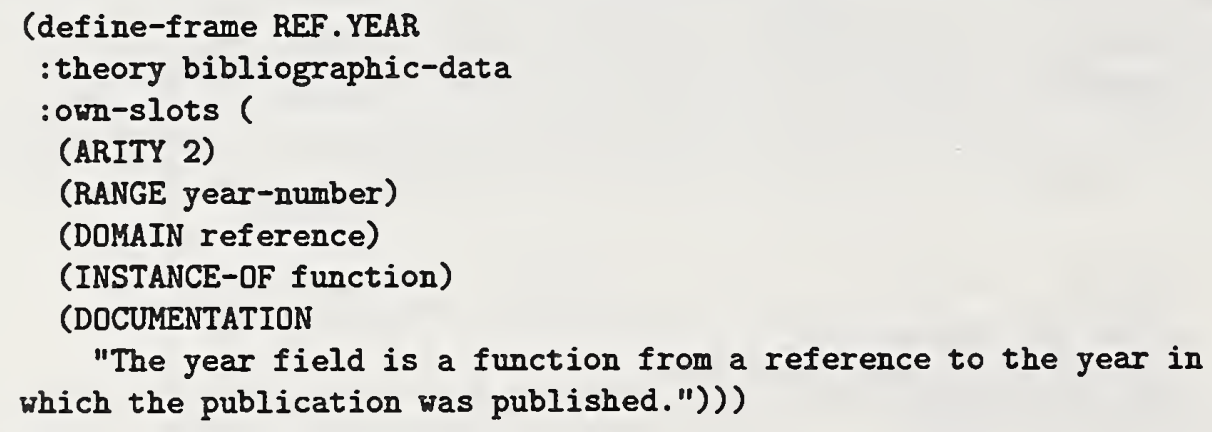

or

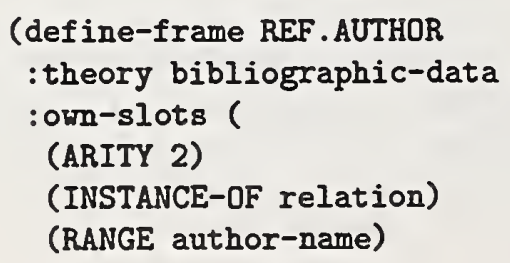




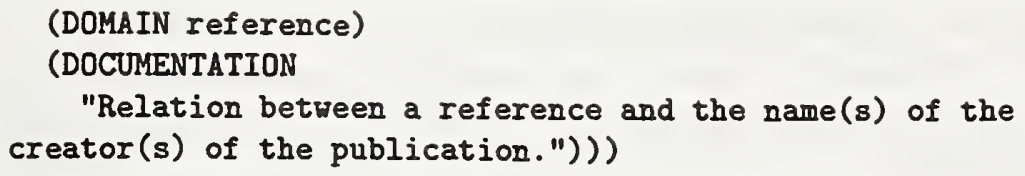

It took me a long time to feel that I had come to an understanding of what this meant. In fact, the translation of the Frame ontology was explicitly undertaken in order to attempt to solve this.

Eventually I came to the conclusion that the explanation lay in set theory, where a Frame Relation effectively defines a set of that 'name', and similarly for Class and Function. The INSTANCE-OF syntax is then a set membership declaration. So, for example, INSTANCE-OF relation

can be read as "this is a member of the set "relation"'.

EXPRESS also has similar notions, in that Schema, Entity and so on are sets [Wil96c]. A declaration like ENTITY AnEntity; specifies that AnEntity is both a set named AnEntity and is a member of the set Entity.

\section{Basic elements of Frame and EXPRESS}

In broad terms both Frame and EXPRESS have three basic semantic elements, although the syntactical representations of these are different.

Frame

1. Theory - the collection of definitions for a particular ontology.

2. Relation - a definition (specification of real world thing of interest).

3. Axiom - a logical expression (or sentence) applied to relation(s). EXPRESS

1. Schema - the collection of definitions for (a cohesive part of) a particular model.

2. Entity - a definition (specification of a real world thing of interest).

3. Constraint - A logical expression (or statement) applied to entity(ies).

Thus, at a broad level, Frame and EXPRESS deal with the same semantic elements; as is often the case, though, the devil is in the details.

Frame makes distinctions between unary relations, which are termed classes, relations where the value one argument is completely defined by the values of the other arguments, which are termed classes, and general n-ary relations which are called relations. 
EXPRESS syntactically has entities and types, where a type can be thought of as a very restricted form of an entity. Effectively a Frame class maps to (the name of) an entity or type. A Frame function can typically be mapped to an entity with a derived attribute. General relations map to entities.

As we have already noted, a Frame theory and an EXPRESS schema are virtually synonymous, and so are Frame relations and EXPRESS entities. The major difference is between Frame axioms and EXPRESS constraints.

In Frame, an axiom is a logical sentence involving relations. These sentences may use the typical forms of predicate logic (e.g., 'there exists' or 'for all' and so on). In EXPRESS a constraint is also specified by a logical statement, but there is no built in language equivalent to 'for all' and friends. Instead, EXPRESS provides a programming language which enables the equivalent to 'for all', etc., to be stated. The programming language includes looping constructs, case statements and so forth. It is therefore often much easier to specify constraints in EXPRESS than in Frame, as in the latter case one is restricted to only using a series of logical sentences, whereas in the forming both general programming techinques are available in addition to logical expressions. That is, often the programming constructs provide a short, elegant and understandable means of specifying the variables and their value ranges that are used in the final constraint statements, whereas in general this is much harder to do when purely restricted to logic - not impossible, but readability is likely to suffer with no increase in either precision or formality.

\section{Conclusions and further work}

There was little difficulty in transliterating Frame ontologies into EXPRESS models, and then further mapping these into idiomatic EXPRESS code. The transliteration process could be reasonably automated, but not the idiomatic mapping.

Further investigation into the use of instances in the Frame language and their mapping relationship to EXPRESS may be required. In the ontologies mapped so far, these have not caused any problems, but it is possible that they might do so in more complex ontologies.

It is unclear to me whether it is possible to map EXPRESS models into the Frame language without losing information. In order to shed some light on this problem I suggest that an experienced Frame (or KIF) modeler map the EXPRESS model given in the appendix (A) into the Frame language. The original scope statement for this model is given in ISO TR9007 [ISO87]. This ISO report describes requirements for conceptual languages and presents the model represented in a variety of generic modeling language types.

\section{References}

[GF92] M. R. Genesereth and R. E. Fikes. Knowledge Interchange Format, Version 3.0 
Reference Manual. Report Logic-92-1, Computer Science Department, Stanford University, June 1992.

[Gin91] M. L. Ginsberg. Knowledge Interchange Format: The KIF of Death AI Magazine, 12(3):57-63, Fall 1991.

[ISO87] ISO TR9007. Information processing systems - Concepts and terminology for the conceptual schema and the information base, 1987.

[ISO94] ISO 10303-11:1994. Industrial automation systems and integration - Product data representation and exchange - Part 11: Description methods: The EXPRESS language reference manual, 1994.

[SW94] Douglas A. Schenck and Peter R. Wilson. Information Modeling the EXPRESS Way. Oxford University Press (ISBN 0-19-308714-3), 1994.

[Wil96a] Peter R. Wilson. Bibliographic Ontology - An EXPRESS Rendition of a Frame Model, February 1996. (Draft).

[Wil96b] Peter R. Wilson. Component Modeling Ontologies - An EXPRESS Rendition of a Frame Model, March 1996. (Draft).

[Wil96c] Peter R. Wilson. EXPRESS and Set Theory, June 1996. (Draft).

[Wil96d] Peter R. Wilson. Frame Ontology - An EXPRESS Rendition of a Frame Model, April 1996. (Draft).

[X395] X3T2 KIF AHG. Knowledge Interchange Format Reference Manual, March 1995. 


\section{A EXPRESS example model}

This Appendix contains a complete and documented EXPRESS model together with an EXPRESS-G graphical version. The model is documented in a similar manner to the STEP models.

\section{A.1 Scope}

The model has to do with the registration of cars and is limited to the scope of interest of the Registration Authority. This Authority exists for the purpose of:

- Knowing who is or was the registered owner of a car at any time from construction to destruction of the car;

- To monitor laws regarding the transfer of ownership of cars;

- To monitor laws regarding the fuel consumption of cars;

- To monitor laws regarding manufacturers of cars.

\section{A.2 Model overview}

The model is described using both EXPRESS and EXPRESS-G. The EXPRESS definitions are primary and the EXPRESS-G diagrams are to assist in understanding the primary model. If there is any conflict between the EXPRESS and EXPRESS-G, then the EXPRESS takes precedence.

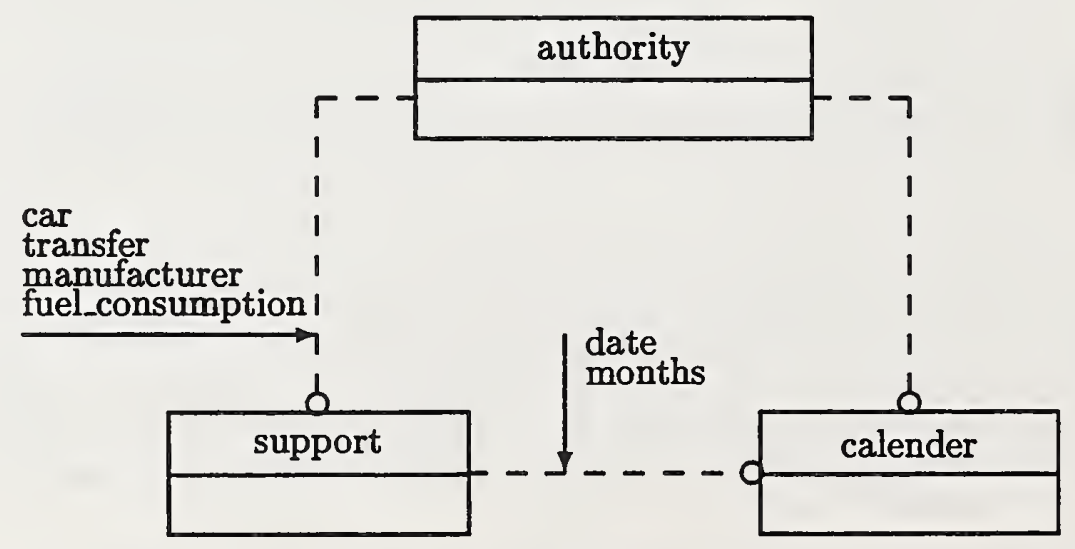

Figure 1: Complete schema-level model for Registration Authority example (Page 1 of 1 ). 
The model consists of three schemas, as shown in figure 1. The schema authority is the primary schema. It references items from the two ancilliary schemas, namely support and calendar. The support schema also references items from the calendar schema.

\section{A.3 Authority schema}

This schema is the primary one in the model and is principally concerned with the main functions of the Registration Authority.

The schema imports definitions from two sources, namely the support and the calendar schemas.

Figure 2 is an EXPRESS-G complete entity-level model for this schema. EXPRESS specification:

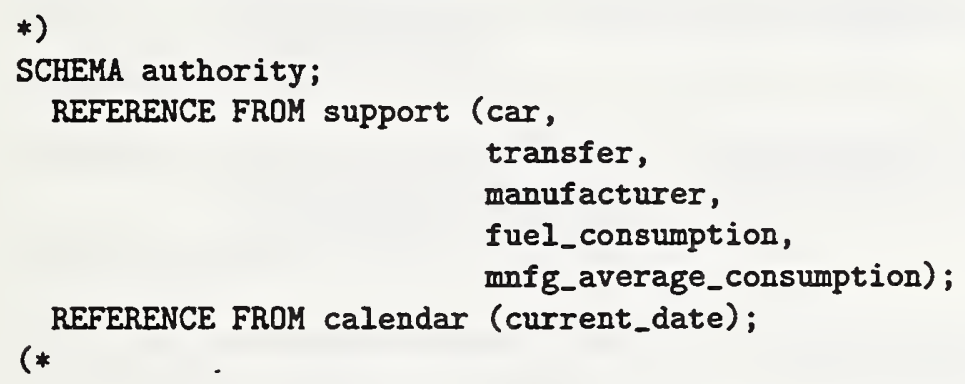

\section{A.3.1 Entity definitions}

\section{Entity HISTORY}

A history records the transfers of ownership of a car over its lifetime. A history must be kept for a certain period after the car is destroyed, after which the ownership records may be destroyed.

EXPRESS specification:

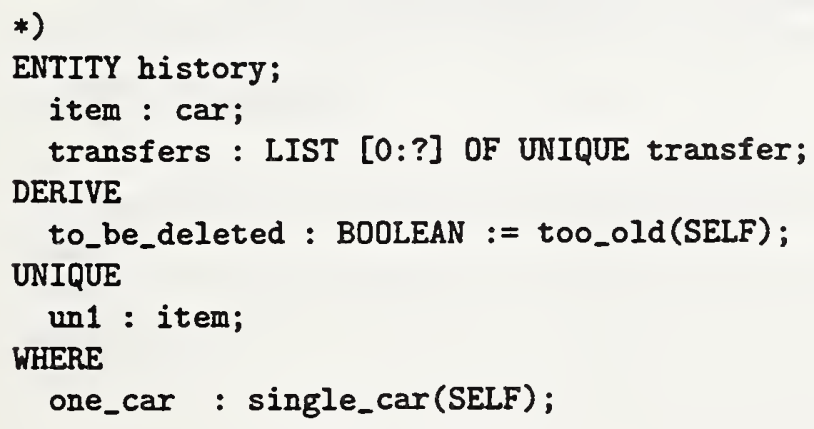



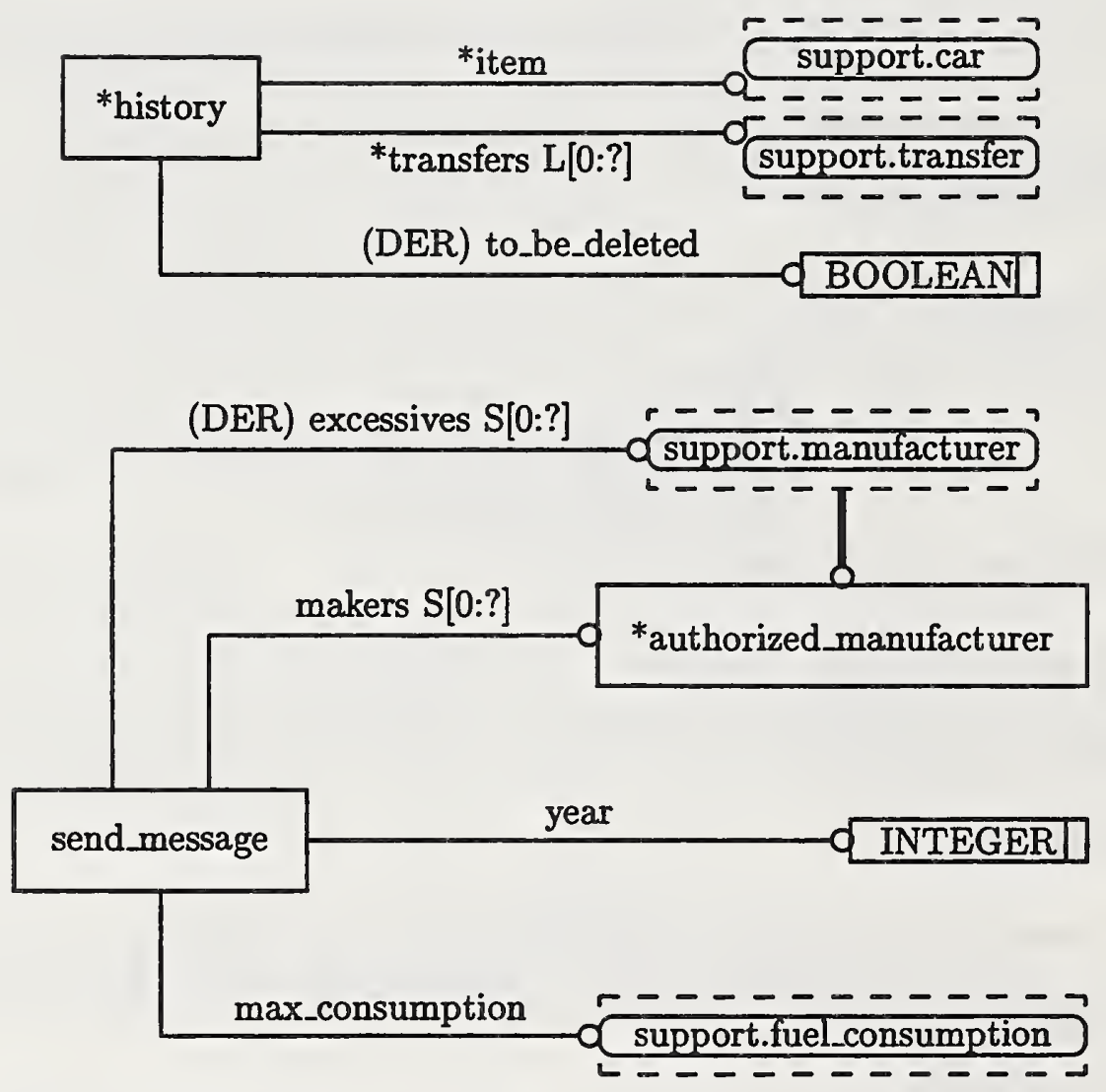

Figure 2: Complete entity-level model of the Authority schema (Page 1 of 1). 


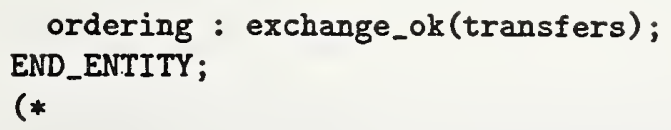

Attribute definitions:

item: The car whose ownership history is being tracked.

transfers: The ownership transfer records of the item.

to_be_deleted: A flag which indicates that this history record may be deleted because the item has been destroyed (TRUE), or that the record shall not be deleted (FALSE).

Formal propositions:

un1: The value of item shall be unique across all instances of history.

one_car: Each transfer collected in a history shall be of the same car.

ordering: The list of transfer shall be in increasing historical order.

\section{Entity AUTHORIZED MANUFACTURER}

An authorized manufacturer is a manufacturer who has been given permission by the Registration Authority to make cars.

EXPRESS specification:

*)

ENTITY authorized_manufacturer

SUBTYPE OF (manufacturer);

END_ENTITY;

(*

\section{Rule MAX NUMBER}

No more than five authorized manufacturers are permitted at any one time.

\section{EXPRESS specification:}

*)

RULE max_number FOR (authorized_manufacturer);

WHERE

max_of_5 : SIZEOF (authorized_manufacturer) $<=5$;

END_RULE;

(* 
Formal propositions:

max_of_5: The rule is violated if there are more than five authorized manufacturers at any time.

\section{Entity SEND MESSAGE}

In January each year the Registration Authority shall send a message to each manufacturer whose cars' average fuel consumption exceeds a certain limit, which may vary from year to year.

\section{EXPRESS specification:}

*)

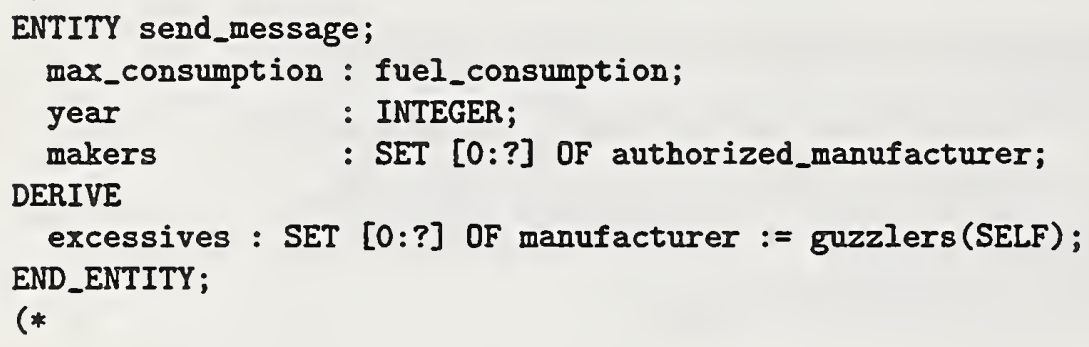

Attribute definitions:

max_consumption: The legal maximum average fuel consumption.

year: The year for which the max consumption value applies.

makers: The authorized manufacturers operating during the year.

excessives: The manufacturers whose cars exceed the consumption limit.

\section{A.3.2 Function and procedure definitions}

\section{Function GUZZLERS}

This function returns the set of manufacturers whose cars exceed an average fuel consumption limit.

Argument definitions:

par: An instance of a send message entity.

RESULT: A set of instances of manufacturer whose cars' average fuel consumption is excessive. 


\section{EXPRESS specification:}

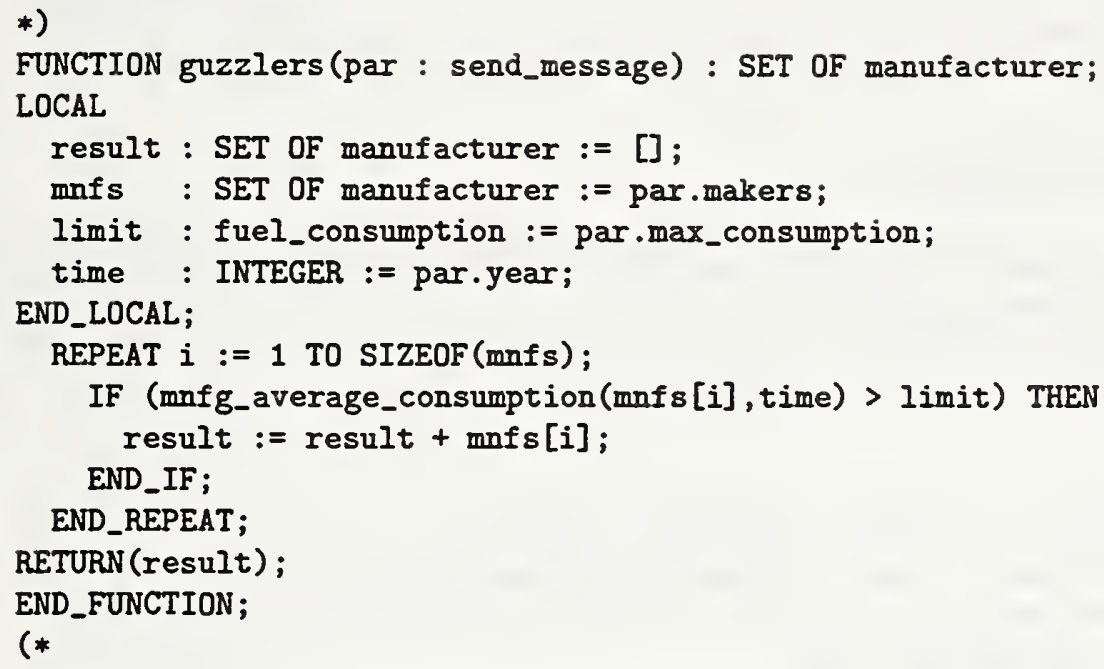

\section{Function TOO OLD}

This function calculates whether the car in a history was destroyed more than two years ago.

Argument definitions:

par: An instance of a history.

RESULT: A Boolean value. TRUE if the car in the input history was destroyed two or more years ago; otherwise FALSE.

\section{EXPRESS specification:}

*)

FUNCTION too_old(par : history) : BOOLEAN;

(* The function returns TRUE if the input history is outdated. That is, if it is of an item that was destroyed more than 2 years ago.*)

IF ('SUPPORT.DESTROYED_CAR' IN par.item) THEN

IF (current_date.year-par.item.destroyed_on.year >= 2) THEN RETURN (TRUE); END_IF;

END_IF;

RETURN (FALSE);

END_FUNCTION;

(* 


\section{Function EXCHANGE OK}

This function checks whether or not the transfers in a list are ordered.

Argument definitions:

par A list of transfer instances.

RESULT A Boolean value. TRUE if the recipient in the $N^{\text {th }}$ transfer is the same as the giver in the $(N+1)^{\text {th }}$ transfer.

\section{EXPRESS specification:}

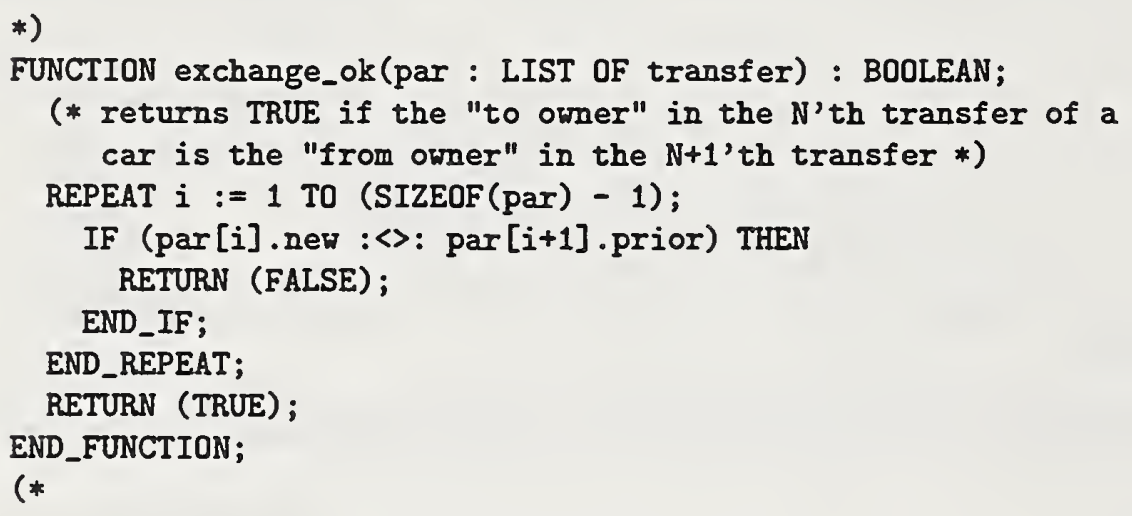

\section{Function SINGLE CAR}

This function checks whether or not the car in a transfer history is the same car specified in each individual transfer.

Argument definitions:

par: A history instance.

RESULT: A Boolean value. TRUE if the history and all its transfers are of the same car, otherwise FALSE.

\section{EXPRESS specification:}

*)

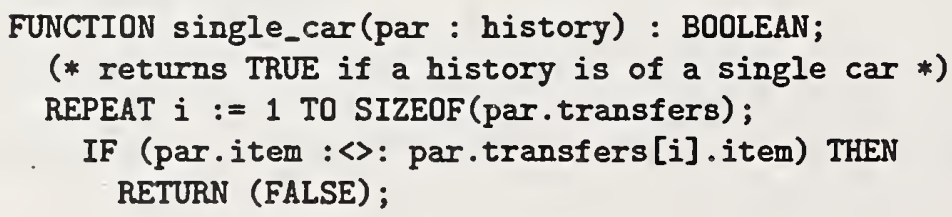




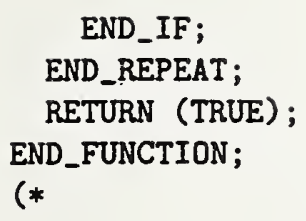

\section{A.3.3 Entity classification structure}

The following indented listing shows the entity classification structure. Entities in upper case characters are defined in this schema. Entities in lower case characters are defined in other schemas.

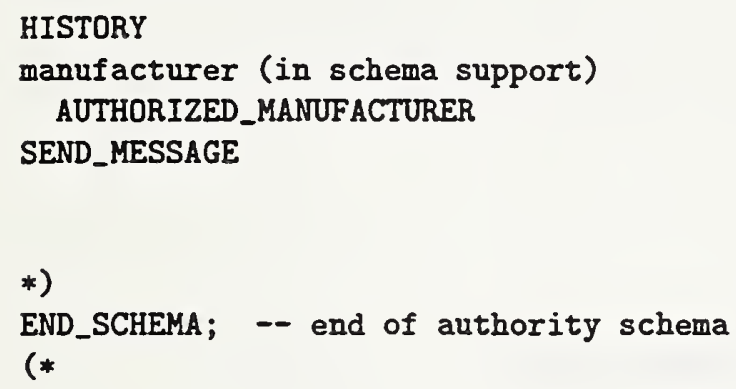

\section{A.4 Support schema}

This schema contains supporting definitions for the primary authority schema.

An EXPRESS-G model of the contents of this schema is given in figure 3 and in figure 4.

The schema imports definitions from the calendar schema.

\section{EXPRESS specification:}

*)

SCHEMA support;

REFERENCE FROM calendar (date, months, days_between); (*

\section{A.4.1 Type definitions}

\section{Type NAME}

The 'name' of something. A human interpretable name which may identify some object, thing or person, etc. For example, Widget Company, Inc..

EXPRESS specification: 
*)

TYPE name = STRING;

END_TYPE;

(*

\section{Type IDENTIFICATION NO}

A character string which may be used as the 'identification number' for a particular instance of some object. This is typically a mixture of alphanumeric characters and other symbols. For example, D20-736597WP23.

EXPRESS specification:

*)

TYPE identification_no = STRING;

END_TYPE;

(*

Type FUEL CONSUMPTION

A measure of the fuel consumption of some powered device.

EXPRESS specification:

*)

TYPE fuel_consumption = REAL;

WHERE

range : $\{4.0<$ SELF $<25.0\}$;

END_TYPE;

(*

Formal propositions:

range: The value is limited to lie in the range 4 to 25 inclusive.

\section{A.4.2 Entity definitions}

Entity TRANSFER

A record of a transfer of a car from one owner to a new owner.

EXPRESS specification:

*)

ENTITY transfer; 

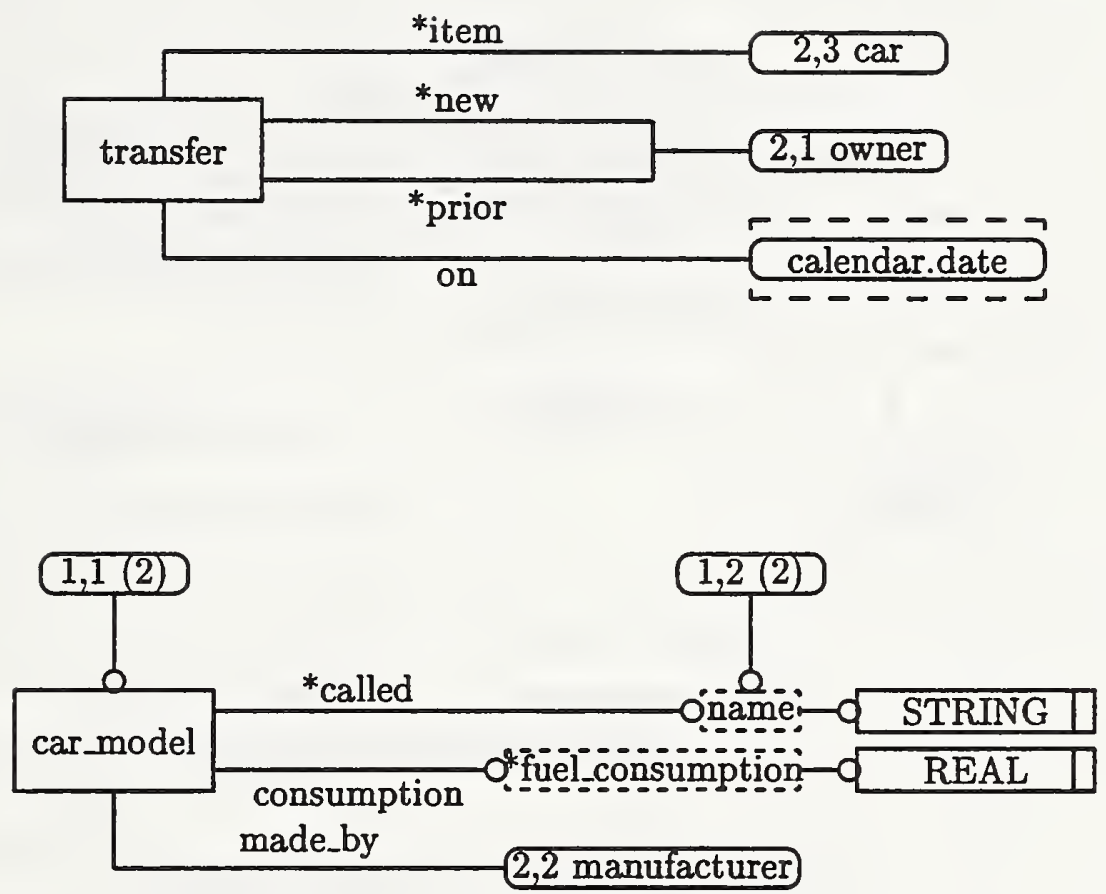

(1,3(2) - id identificationno:- Q STRING

Figure 3: Complete entity-level model of the Support schema (Page 1 of 2). 

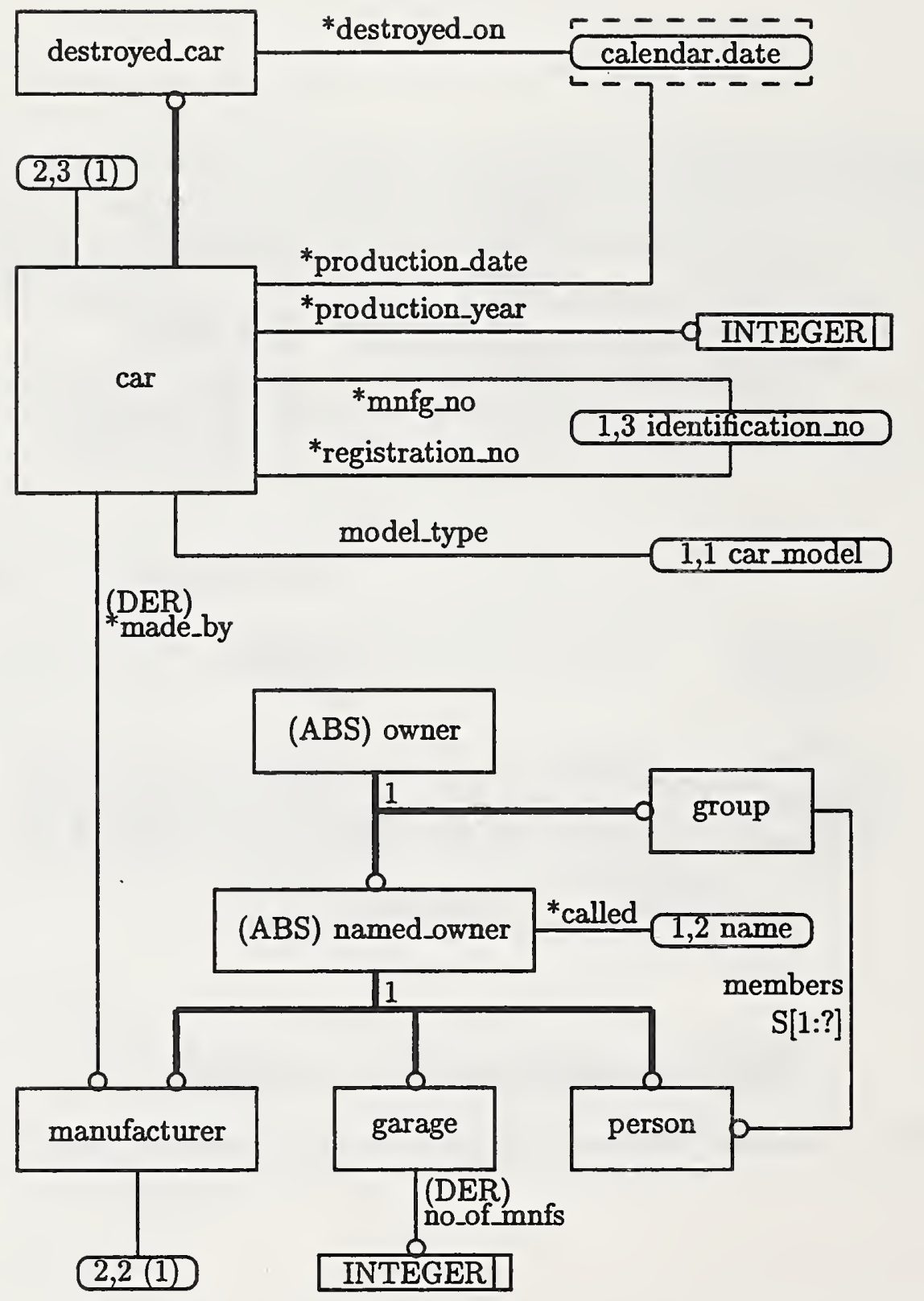

Figure 4: Complete entity-level model of the Support schema (Page 2 of 2). 


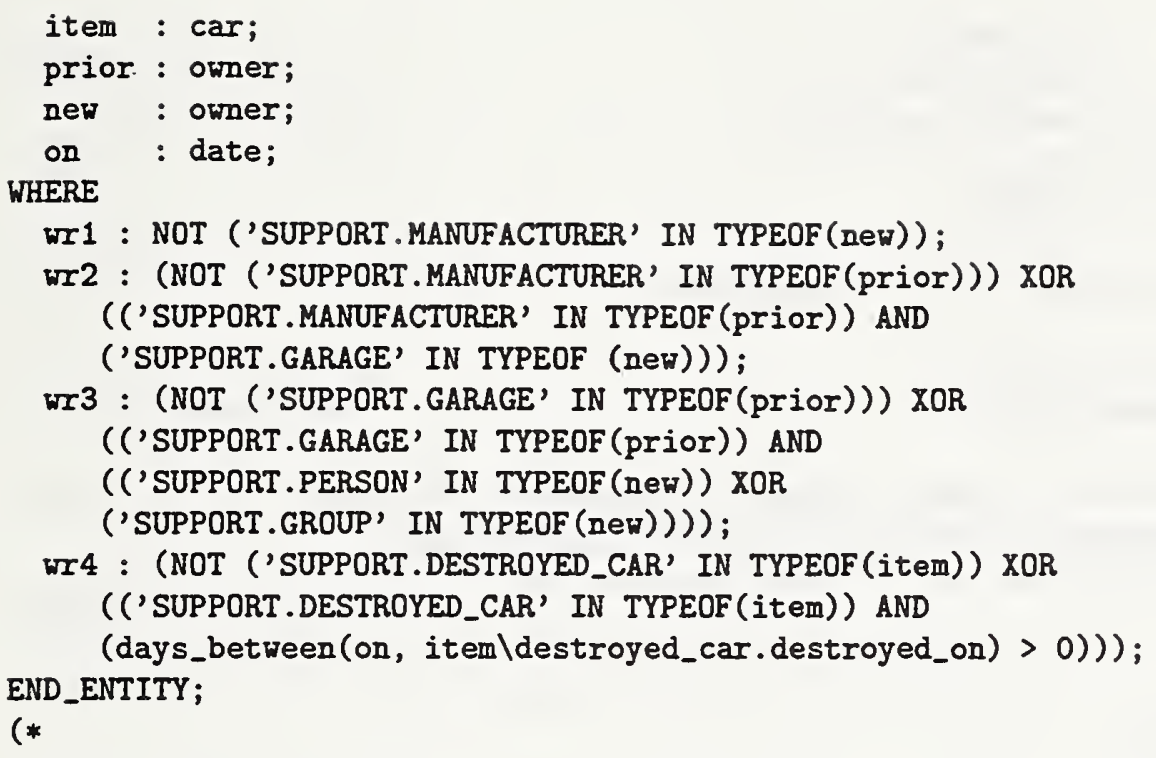

Attribute definitions:

item: The car being transferred.

prior: The prior owner of the item.

new: The new owner of the item.

on: The date of the transfer.

Formal propositions:

wr1: A car cannot be transferred to a manufacturer.

wr2: A manufacturer can only transfer a car to a garage.

wr3: A garage can only transfer a car to either a person of a group of people.

wr4: A car which has been destroyed cannot be transferred.

\section{Entity CAR}

A car.

EXPRESS specification:

*)

ENTITY car; 


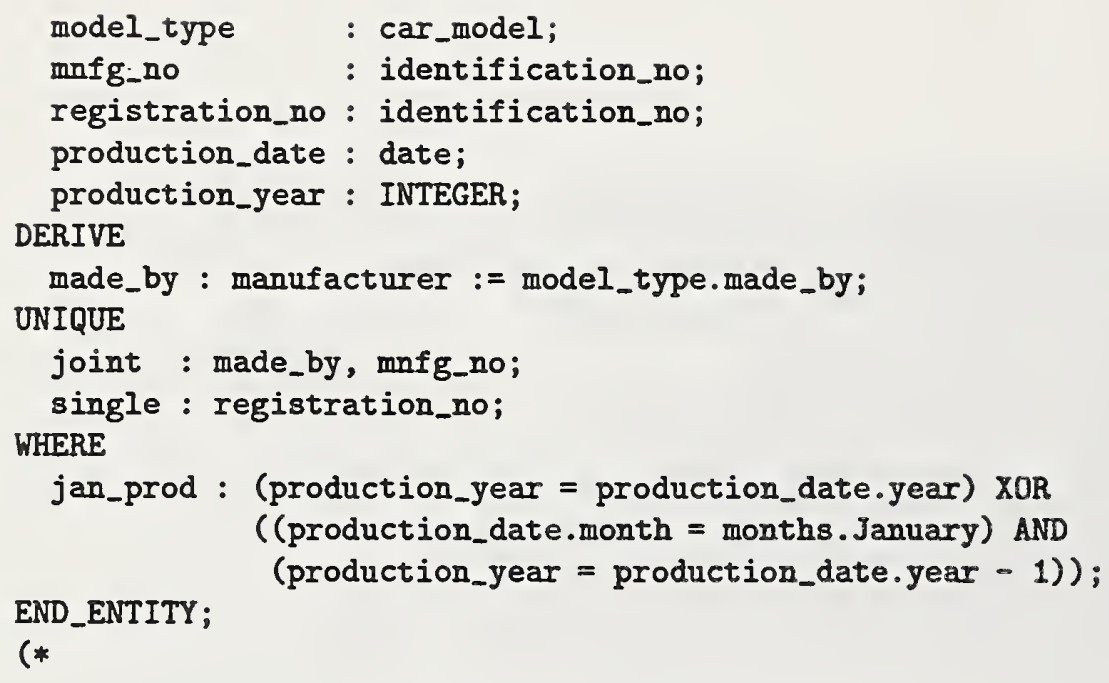

Attribute definitions:

model_type: The car model.

mnfg_no: An identification number of the car assigned by the car's manufacturer.

registration_no: An identification number for the car assigned by the Registration Authority.

production_date: The date on which the car was produced.

production_year: The registered year of production of the car.

made_by: The manufacturer of the car.

Formal propositions:

joint: The mnfg no given to a car is unique for the given car manufacturer.

single: Each car is given a unique registration no by the Registration Authority.

jan_prod: The registered production year is the same as the year in which the car was produced, except that cars produced in January may be registered as having been produced in the previous year.

\section{Entity DESTROYED CAR}

A car may be destroyed, in which case its date of destruction is recorded. EXPRESS specification: 


\section{*)}

ENTITY destroyed_car

SUBTYPE OF (car);

destroyed_on : date;

WHERE

dates_ok : days_between(production_date, destroyed_on) $>=0$; END_ENTITY;

(*

Attribute definitions:

destroyed_on: The date on which the car was destroyed.

Formal propositions:

dates_ok: A car cannot be destroyed before it has been made.

Entity CAR MODEL

A particular type of car.

EXPRESS specification:

*)

ENTITY car_model;

called : name;

made_by : manufacturer;

consumption : fuel_consumption;

UNIQUE

un1 : called;

END_ENTITY;

(*

Attribute definitions:

called: The name of the model.

made_by: The manufacturer of the model.

consumption: The average fuel consumption of all cars of this model type.

Formal propositions:

un1: Each car model has a distinct name. 


\section{Entity OWNER}

An owner of a car. Owners are categorized into named owner and group. EXPRESS specification:

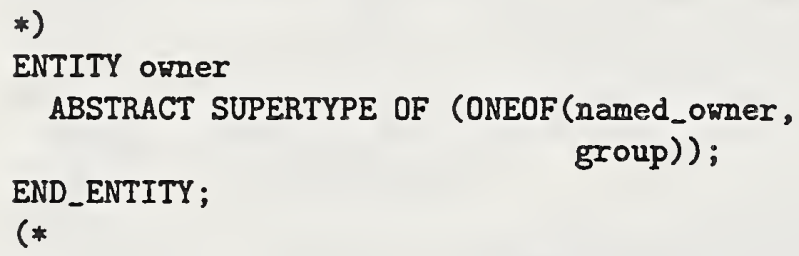

\section{Entity NAMED OWNER}

An owner who has a name. These are categorized into manufacturer, garage and person. EXPRESS specification:

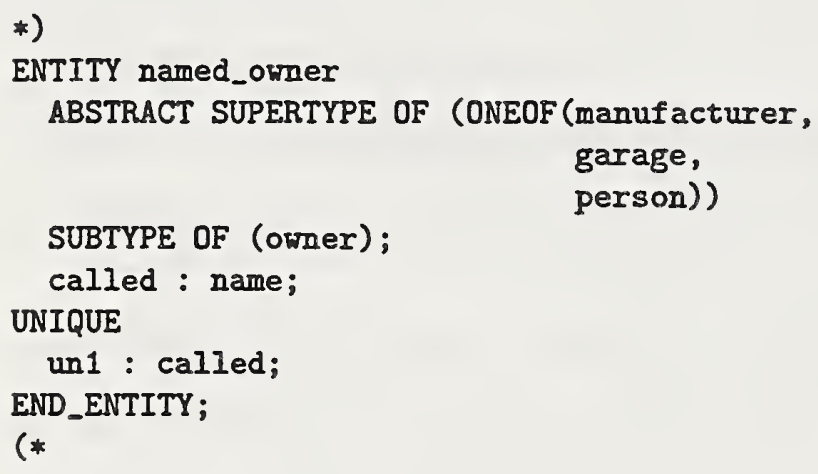

Attribute definitions:

called: The name of the owner.

Formal propositions:

un1: Owner's names are unique.

\section{Entity MANUFACTURER}

A type of named car owner. Manufacturers may also manufacture cars. EXPRESS specification:

*)

ENTITY manufacturer 


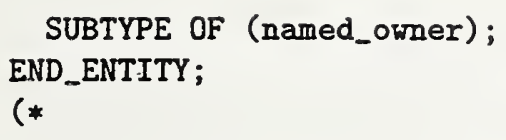

\section{Entity GARAGE}

A type of named car owner.

\section{EXPRESS specification:}

\section{*)}

ENTITY garage

SUBTYPE OF (named_owner);

DERIVE

no_of_mnfs : INTEGER := dealer_for_mnfs(SELF);

WHERE

wr1 : $\{1<=$ no_of_mnfs $<=3\}$;

END_ENTITY;

(*

Attribute definitions:

no_of_mnfs: The number of different manufacturers of the cars owned by the garage.

Formal propositions:

wr1: At any particular time, a garage shall not own cars made by more than three manufacturers.

\section{Entity PERSON}

A type of named car owner.

\section{EXPRESS specification:}

\section{*)}

ENTITY person

SUBTYPE OF (named_owner);

END_ENTITY;

(*

\section{Entity GROUP}

A type of car owner consisting of a group of people.

EXPRESS specification: 
*)

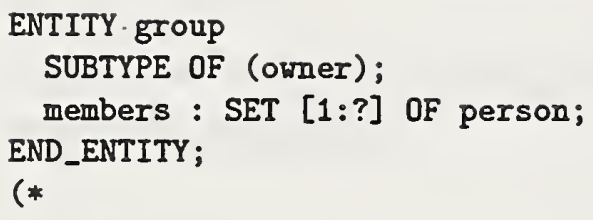

Attribute definitions:

members: The people who form the group.

\section{A.4.3 Function and procedure definitions}

\section{Function DEALER FOR MNFS}

This function calculates the total number of distinct manufacturers of cars owned by a garage.

Argument definitions:

dealer: An instance of a garage.

RESULT: The number of distinct manufacturers of the cars owned by the garage.

EXPRESS specification:

*)

FUNCTION dealer_for_mnfs(dealer : garage) : INTEGER;

LOCAL

cars : SET OF car := [];

transfers : SET OF transfer := [];

makers : SET OF manufacturer := [];

END_LOCAL;

transfers := USEDIN (dealer, 'TRANSFER.NEW');

REPEAT $i:=1$ TO SIZEOF(transfers);

cars := cars + transfers [i].item;

END_REPEAT;

transfers := USEDIN(dealer, 'TRANSFER.PRIOR');

REPEAT $i:=1$ TO SIZEOF (transfers);

cars := cars - transfers $[i]$.item;

END_REPEAT;

REPEAT $i:=1$ TO SIZEOF (cars);

makers := makers + cars [i].model_type.made_by;

END_REPEAT;

RETURN (SIZEOF (makers));

END_FUNCTION;

(* 


\section{Function MNFG AVERAGE CONSUMPTION}

This function calculates the average fuel consumption in a given year of all the cars made by a particular manufacturer.

Argument definitions:

mnfg: A manufacturer.

when: An INTEGER representing a particular year.

RESULT: A REAL giving the average fuel consumption of the manufacturer's cars during a particular year.

EXPRESS specification:

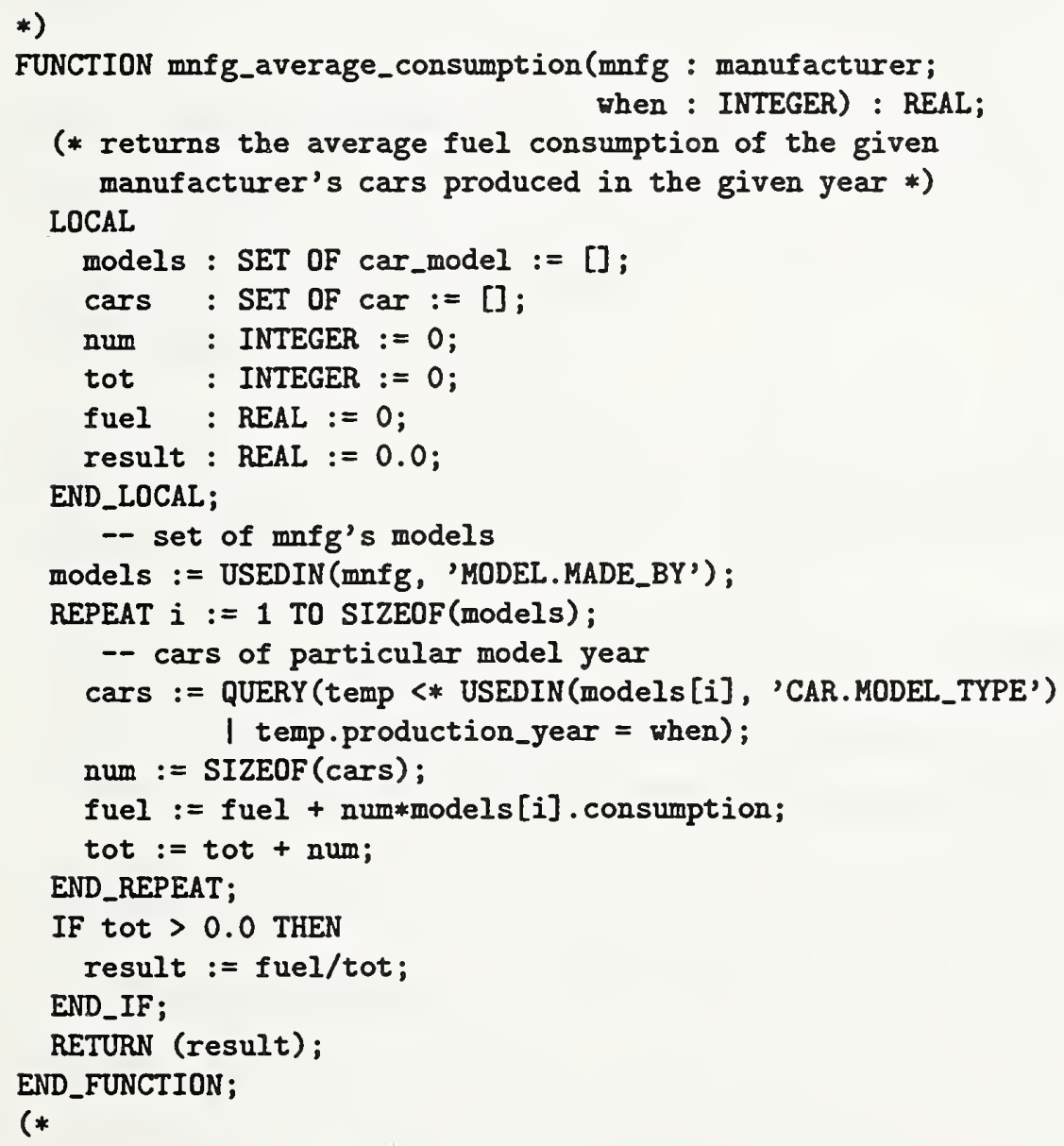




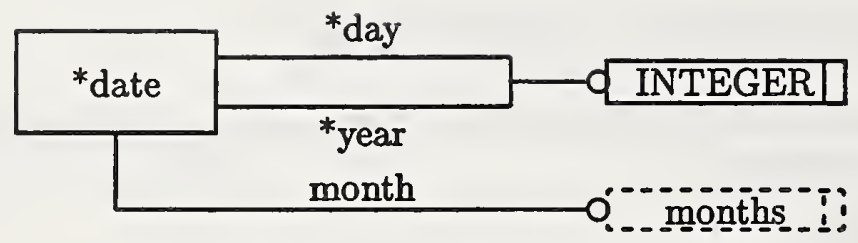

Figure 5: Complete entity-level model of Calendar schema (Page 1 of 1).

\section{A.4.4 Entity classification structure}

The following indented listing shows the entity classification structure. Entities in upper case characters are defined in this schema. Entities in lower case characters are defined in other schemas.

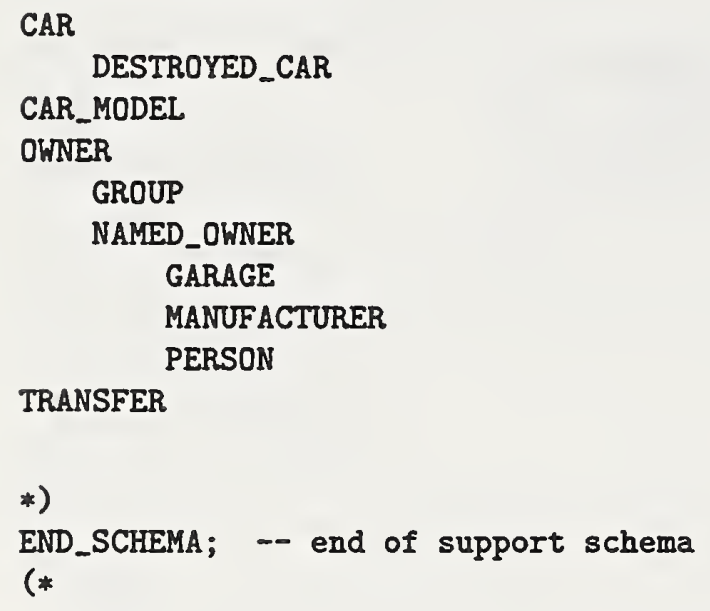

\section{A.5 Calendar schema}

This schema contains definitions related to dates and other calendrical items.

Figure 5 is an EXPRESS-G model showing the contents of this schema. EXPRESS specification:

\section{*)}

SCHEMA calendar;

(* 


\section{A.5.1 Type definitions}

\section{Type MONTHS}

An enumeration of the months of the year. January is the first month in a year and December is the last month in a year.

EXPRESS specification:

*)

TYPE months = ENUMERATION OF

(January, February, March,

April, May, June,

July, August, September,

October, November, December);

END_TYPE;

(*

\section{A.5.2 Entity definitions}

\section{Entity DATE}

A date $\mathrm{AD}$ in the Gregorian calendar.

EXPRESS specification:

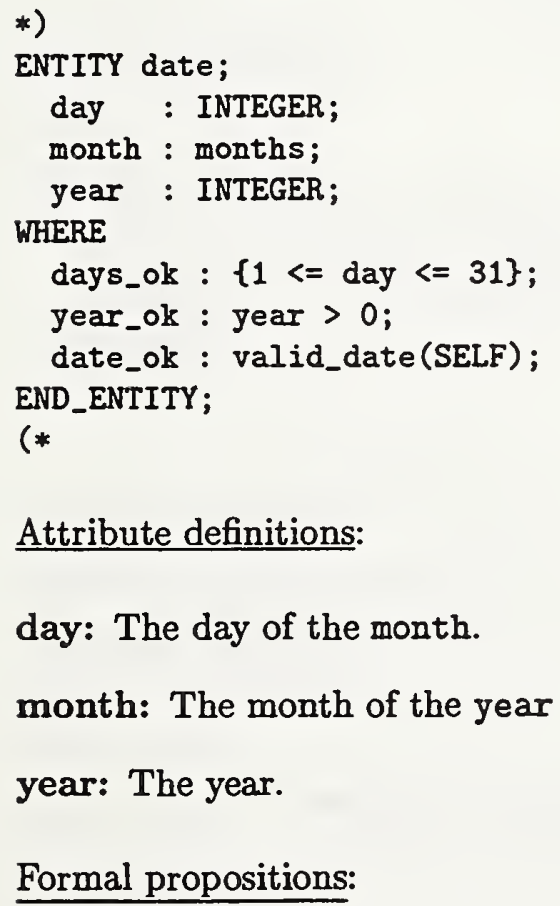

Attribute definitions:

day: The day of the month.

month: The month of the year

year: The year.

Formal propositions: 
days_ok: The day shall be numbered between 1 and 31 inclusive.

year_ok: The year shall be greater than zero.

date_ok: The combination of day, month and year shall form a valid date, taking into account the differing numbers of days in particular months, and also the effect of leap years.

\section{A.5.3 Function and procedure definitions}

\section{Function VALID DATE}

This function checks a date for valid day, month, year combinations.

Argument definitions:

par: A date.

RESULT: A Boolean. TRUE if the date has a valid day, month, year combination, FALSE otherwise.

\section{EXPRESS specification:}

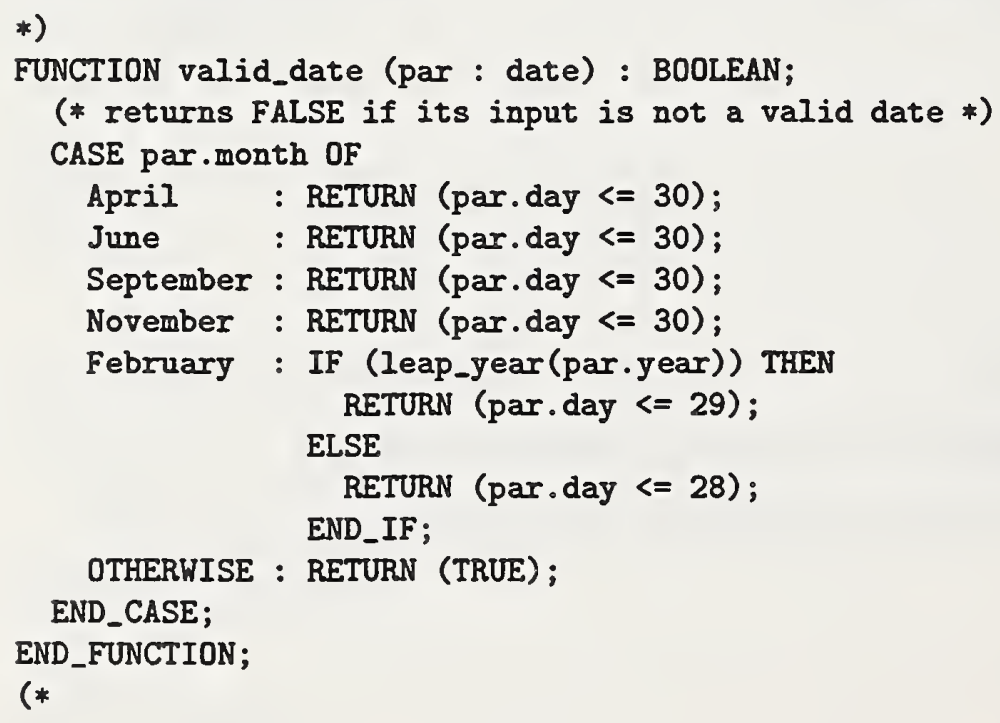

\section{Function LEAP YEAR}

This function checks whether a given integer could represent a leap year.

Argument definitions: 
year: An INTEGER.

RESULT: A Boolean. TRUE if year is a leap year, otherwise FALSE.

EXPRESS specification:

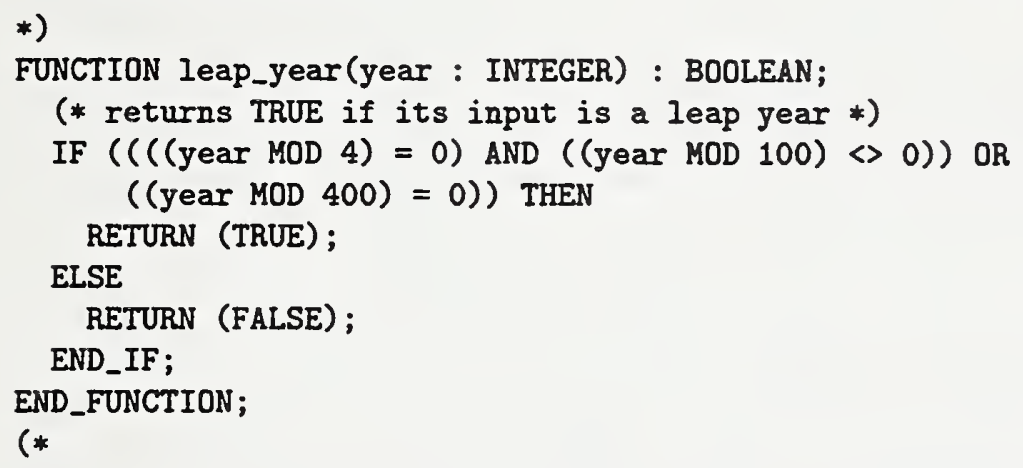

\section{Function CURRENT DATE}

This function returns the current date.

Argument definitions:

RESULT: The current date.

EXPRESS specification:

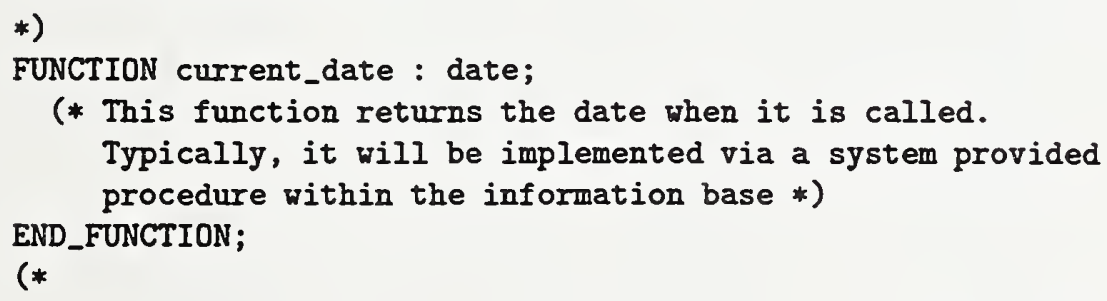

\section{Function DAYS BETWEEN}

This function returns the number of days between any two dates.

Argument definitions:

d1: A date.

d2: A date. 
RESULT: An Integer. The number of days between the two input dates. If d1 is earlier than $d 2$ a positive integer is returned; if $d 1$ is later than $d 2$ a negative integer is returned; otherwise zero is returned.

\section{EXPRESS specification:}

*)

FUNCTION days_between(d1, d2 : date) : INTEGER;

(* returns the number of days between two input dates. If d1

is earlier than d2, a positive number is returned. *)

END_FUNCTION;

(*

\section{A.5.4 Entity classification structure}

The following indented listing shows the entity classification structure. Entities in upper case characters are defined in this schema. Entities in lower case characters are defined in other schemas.

DATE

*)

END_SCHEMA; -- end of calendar schema

(* 



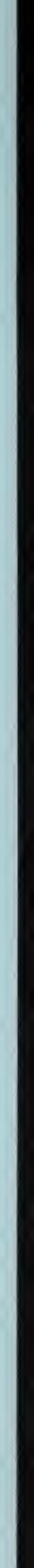

